\title{
Pressure and volume in the first law of black hole thermodynamics
}

\author{
Brian P. Dolan \\ Department of Mathematical Physics, National University of Ireland, \\ Maynooth, Ireland \\ and \\ Dublin Institute for Advanced Studies, 10 Burlington Rd., Dublin, Ireland \\ e-mail: bdolan@thphys.nuim.ie
}

November 14, 2011 


\begin{abstract}
The mass of a black hole is interpreted, in terms of thermodynamic potentials, as being the enthalpy, with the pressure given by the cosmological constant. The volume is then defined as being the Legendre transform of the pressure and the resulting relation between volume and pressure is explored in the case of positive pressure. A virial expansion is developed and a van der Waals like critical point determined. The first law of black hole thermodynamics includes a $P d V$ term which modifies the maximal efficiency of a Penrose process. It is shown that, in four dimensional space-time with a negative cosmological constant, an extremal charged rotating black hole can have an efficiency of up to $75 \%$, while for an electrically neutral rotating back hole this figure is reduced to $52 \%$, compared to the corresponding values of $50 \%$ and $29 \%$ respectively when the cosmological constant is zero.
\end{abstract}

PACS nos: 04.60.-m; 04.70.Dy 


\section{Introduction}

The thermodynamics of black holes is a rich and fascinating area of research which continues to yield surprises. The first law of black hole thermodynamics is usually written as

$$
d M=T d S+\Omega d J+\Phi d Q
$$

where $T=\frac{\kappa}{2 \pi}$ is the Hawking temperature of the black hole (with $\kappa$ the surface gravity), $S=\frac{A}{4}$ the entropy (with $A$ the area in Planck units), $\Omega$ the angular velocity, $J$ the angular momentum, $\Phi$ the electrostatic potential difference between infinity and the horizon, $Q$ the electric charge and $M$ the mass. The mass is usually interpreted as the internal energy, in the thermodynamic sense, of the black hole, but it was suggested in [1] that it is more correctly interpreted as the enthalpy. In this context it is notable that there is no $P d V$ term in (1), corresponding to a change in volume at ambient pressure $P$. When a cosmological constant, $\Lambda$, is included there is a natural candidate for a pressure, $P=-\frac{\Lambda}{8 \pi}$, and it was proposed in [2] that the volume of the black hole be defined as the thermodynamic variable conjugate to $P$. Interpreting the mass as the enthalpy, equation (1) should then be modified to

$$
d M=T d S+V d P+\Omega d J+\Phi d Q
$$

where the thermodynamic volume is defined to be $V=\left.\frac{\partial M}{\partial P}\right|_{S, J, Q}$, [1]. The idea that $\Lambda$ should be thought of as a thermodynamic variable that can be varied is not new and has been considered by a number of authors, [3]- [9]. Equation (2), with $Q=0$, was studied in [8], in the context of varying $\Lambda$, without any particular physical interpretation being given to the thermodynamic conjugate of $\Lambda$, often denoted $\Theta$.

One may question whether it is appropriate to identify $\Lambda$ with a thermodynamic pressure. While a cosmological constant gives a pressure term in Einstein's equations, a fluid dynamical pressure is not necessarily the same as a thermodynamic pressure. In equilibrium situations however it is presumably correct to identify the fluid dynamical pressure with the thermodynamic pressure and we shall do so here.

In general the enthalpy, $H$, is the heat energy beloved of chemists, it is not the internal energy, $U$, of the first law of thermodynamics. That distinction goes to the Legendre transform of the enthalpy,

$$
U=H-P V
$$


where $H$ is a function of $S, P, J$ and $Q$ while $U(S, V, J, Q)$ is a function of purely extensive variables. Then we get the usual form of the first law,

$$
d U=T d S-P d V+\Omega d J+\Phi d Q .
$$

This equation was written down in [9], using $\Theta-\Lambda$ notation, but its consequences were not pursued.

When $\Lambda$ is non-zero we should expect the $P d V$ term to contribute to the mechanical energy that can be extracted from a black hole, by a Penrose process for example. For a negative $\Lambda$ (positive pressure) the $P d V$ term gives a positive contribution to $d U$ if the black hole shrinks, and the $P d V$ term reduces the amount of energy available for extraction as mechanical work $W$, with $d W=-d U$, hence reducing the efficiency. However we shall show that the maximal efficiency actually increases, relative to the $\Lambda=0$ case, when $\Lambda<0$, because the maximal angular momentum of a black hole in AdS is greater than that of one with $\Lambda=0$ and this can outweigh the reduction in efficiency: the engine may not be as efficient at a given $J$ but it can be pushed to higher $J$. Conversely one would expect that, for a positive $\Lambda$ more energy becomes available at a given $J$, relative to $\Lambda=0$, as the black hole shrinks.

Of course there are no pistons pushing against a gas for a black hole, but a negative cosmological constant contributes a negative energy density to space-time so a shrinking black-hole exposes negative energy, thus increasing the black hole's internal energy and decreasing the amount of energy available for mechanical work. Conversely a positive cosmological constant would presumably release extra energy as the black hole shrinks, that can be used to do work, hence increasing the efficiency at a given $J$.

The most efficient way to extract energy from a black hole is in an isentropic process, with $d S=0$ and the area of the event horizon constant. So

$$
d U \geq d U_{\min }=-P d V+\Omega d J+\Phi d Q .
$$

We shall see that, for a rotating black hole, it is possible to reduce $V$ while keeping $S$ constant. The maximum amount of mechanical work that can be extracted in passing from an initial state $i$ to a final state $f$ is

$$
W_{\max }=-\int_{i}^{f} d U_{\min } .
$$


The efficiency is defined to be the ratio of the mechanical energy extracted to the initial heat energy (enthalpy),

$$
\eta=\frac{W_{\max }}{M_{i}}
$$

where the initial enthalpy is identified with the initial mass, $M_{i}$. It will be shown that, for $\Lambda<0$, this can be as high as $52 \%$ for a rotating neutral black hole and $75 \%$ for a charged black hole.

The volume of a black hole has only recently been considered as a thermodynamic variable, [2, 13. At the simplest level, there is a natural tendency to assume that the area and the volume are related geometrically and are not independent. For a Schwarzschild black hole with radius $r_{h}$, for example, the area is of course $4 \pi r_{h}^{2}$ and indeed the thermodynamic volume works out to be $\frac{4 \pi}{3} r_{h}^{3}$, but this seems co-incidental and no particular significance should be attached to it. At a deeper level it is not even clear how to define a volume geometrically, as the metric is not static in the interior of a black hole. For a Schwarzschild black hole which is not rotating we shall see that the thermodynamic volume and the area are not independent: fixing $S$ fixes $V$ so $d V=0$ in an isentropic process, and the $P d V$ term does not contribute to the first law. But for a rotating black hole the area of the event horizon does not determine the thermodynamic volume uniquely and it is possible to vary the volume keeping the entropy constant, by changing the angular momentum and/or the charge.

The properties of the thermodynamic volume and its contribution to the first law of black hole thermodynamics are explored in detail in this paper for a rotating charged black hole in four dimensional space-time with a negative $\Lambda$. In section 2 thermodynamic potentials and the equation of state are discussed and the Legendre transform from the enthalpy to the internal energy is given explicitly. In section 3 the efficiency of a Penrose type process is analysed and section 4 contains a discussion and outlook. Two appendices are dedicated to the technicalities of deriving some results used in the text

\section{The internal energy}

Including a pressure term in the first law gives

$$
d U=T d S-P d V+\Omega d J+\Phi d Q
$$


where the internal energy, $U(S, V, J, Q)$, is a function of extensive variables. The thermodynamic volume $V$ is the conjugate variable to the pressure and is obtained from the mass, which is identified in [1] with the enthalpy, $M=$ $H(S, P, J, Q)$, by

$$
V=\left.\frac{\partial H}{\partial P}\right|_{S, J, Q} .
$$

It was proposed in [2] that (8) be defined to be the thermodynamic volume of the black hole.

The line element for a charged rotating black hole in 4-dimensional anti-de Sitter space is 10 .

$d s^{2}=-\frac{\Delta}{\rho^{2}}\left(d t-\frac{a \sin ^{2} \theta}{\Xi} d \phi\right)^{2}+\frac{\rho^{2}}{\Delta} d r^{2}+\frac{\rho^{2}}{\Delta_{\theta}} d \theta^{2}+\frac{\Delta_{\theta} \sin ^{2} \theta}{\rho^{2}}\left(a d t-\frac{r^{2}+a^{2}}{\Xi} d \phi\right)^{2}$,

where

$$
\begin{aligned}
& \Delta=\frac{\left(r^{2}+a^{2}\right)\left(L^{2}+r^{2}\right)}{L^{2}}-2 m r+q^{2}, \quad \Delta_{\theta}=1-\frac{a^{2}}{L^{2}} \cos ^{2} \theta, \\
& \rho^{2}=r^{2}+a^{2} \cos ^{2} \theta, \quad \Xi=1-\frac{a^{2}}{L^{2}},
\end{aligned}
$$

and the cosmological constant is $\Lambda=-\frac{3}{L^{2}}$, which is related to the pressure by $\frac{1}{L^{2}}=\frac{8 \pi P}{3}$.

The physical properties of this space-time are well known [11, and the first law, applied to this metric, was discussed in [12], but without a $P d V$ term. The metric parameters $m$ and $q$ are related to the mass and charge by

$$
M=\frac{m}{\Xi^{2}}, \quad Q=\frac{q}{\Xi} .
$$

The event horizon, $r_{+}$, lies at the largest root of $\Delta(r)=0$, so

$$
M=\frac{\left(r_{+}^{2}+a^{2}\right)\left(L^{2}+r_{+}^{2}\right)+q^{2} L^{2}}{2 r_{+} L^{2} \Xi^{2}},
$$

and the area of the event horizon is

$$
A=4 \pi \frac{\left(r_{+}^{2}+a^{2}\right)}{\Xi} .
$$


The temperature is

$$
T=\frac{\left(L^{2}+3 r_{+}^{2}\right) r_{+}^{2}-a^{2}\left(L^{2}-r_{+}^{2}\right)-q^{2} L^{2}}{4 \pi L^{2} r_{+}\left(r_{+}^{2}+a^{2}\right)} .
$$

The angular momentum, $J=a M$ and the relevant thermodynamic angular velocity is

$$
\Omega=\frac{a\left(L^{2}+r_{+}^{2}\right)}{L^{2}\left(r_{+}^{2}+a^{2}\right)} .
$$

The electrostatic potential is

$$
\Phi=\frac{q r_{+}}{r_{+}^{2}+a^{2}}
$$

One can scale $L$ out from all the above expressions by defining dimensionless variables $\bar{M}=M / L, \bar{a}=a / L, \bar{r}_{+}=r_{+} / L$, etc., but we prefer to keep $L$ explicit to expose more clearly the rôle of the pressure, and make the comparison with the $L \rightarrow \infty$ limit clear.

Under the assumptions made here the thermodynamic volume for the Kerr-Newman-AdS black hole works out to be

$$
V=\frac{2 \pi}{3}\left\{\frac{\left(r_{+}^{2}+a^{2}\right)\left(2 r_{+}^{2} L^{2}+a^{2} L^{2}-r_{+}^{2} a^{2}\right)+L^{2} q^{2} a^{2}}{L^{2} \Xi^{2} r_{+}}\right\},
$$

which is a simple generalisation of the Kerr-AdS volume derived in [13]. A direct derivation of (17) from (12) is most easily achieved by first writing the mass as a function of $(S, P, J, Q)$, differentiating with respect to $P$, and then transforming back to $\left(r_{+}, a, q, L\right)$.

When $a=0$ the area (13) and volume (17) are not independent and the area determines the volume uniquely, but when $a$ is non-zero the area and the volume become independent.

For asymptotically flat space, with $L \rightarrow \infty$, one has

$$
V=\frac{2 \pi}{3} \frac{\left(r_{+}^{2}+a^{2}\right)\left(2 r_{+}^{2}+a^{2}\right)+q^{2} a^{2}}{r_{+}}
$$

While this reduces to the naïve result, $\frac{4 \pi r_{+}^{3}}{3}$, for the Schwarzschild black hole, a geometrical interpretation when $a$ is non-zero is not so clear.

A correct description of the thermodynamics of the black hole, in terms of thermodynamic potentials, requires replacing the geometric variables $\left(r_{+}, L, a, q\right)$ 
with thermodynamic variables $(S, P, J, Q)$. The relevant expression for the mass, and hence the enthalpy, was derived in [9],

$$
H(S, P, J, Q):=\frac{1}{2} \sqrt{\frac{\left(S+\pi Q^{2}+\frac{8 P S^{2}}{3}\right)^{2}+4 \pi^{2}\left(1+\frac{8 P S}{3}\right) J^{2}}{\pi S}} .
$$

This generalises the Christodoulou-Ruffini formula [14 for the mass of a rotating black hole in terms of its irreducible mass, $M_{i r r}$. The irreducible mass for a black hole with entropy $S$ is the mass of a Schwarzschild black hole with the same entropy, $M_{i r r}^{2}=\frac{S}{4 \pi}$.

In terms of thermodynamic variables the temperature is, [9],

$$
T=\left.\frac{\partial H}{\partial S}\right|_{J, Q, P}=\frac{1}{8 \pi H}\left[\left(1+\frac{\pi Q^{2}}{S}+\frac{8 P S}{3}\right)\left(1-\frac{\pi Q^{2}}{S}+8 P S\right)-4 \pi^{2}\left(\frac{J}{S}\right)^{2}\right]
$$

and the thermodynamic volume is

$$
V=\left.\frac{\partial H}{\partial P}\right|_{S, J, Q}=\frac{2}{3 \pi H}\left[S\left(S+\pi Q^{2}+\frac{8 P S^{2}}{3}\right)+2 \pi^{2} J^{2}\right]
$$

which is manifestly positive.

The Legendre transform $U=H-P V$ gives the thermal energy, a function of purely extensive variables. The transform is evaluated in an appendix to be

$$
\begin{aligned}
U(S, V, J, Q)=\left(\frac{\pi}{S}\right)^{3} & {\left[\left(\frac{3 V}{4 \pi}\right)\left\{\left(\frac{S}{2 \pi}\right)\left(\frac{S}{\pi}+Q^{2}\right)+J^{2}\right\}\right.} \\
& \left.-|J|\left\{\left(\frac{3 V}{4 \pi}\right)^{2}-\left(\frac{S}{\pi}\right)^{3}\right\}^{\frac{1}{2}}\left(\frac{S Q^{2}}{\pi}+J^{2}\right)^{\frac{1}{2}}\right] .
\end{aligned}
$$

Dimensional analysis implies that $U$ is only a function of three independent variables, since $U \rightarrow \lambda U$ when $S \rightarrow \lambda^{2} S, V \rightarrow \lambda^{3} V, J \rightarrow \lambda^{2} J$ and $Q \rightarrow \lambda Q$.

One must be careful taking the $J \rightarrow 0$ limit of these potentials. In this limit the enthalpy

$$
H(S, P, 0, Q)=\frac{1}{2} \sqrt{\frac{S}{\pi}}\left(1+\frac{\pi Q^{2}}{S}+\frac{8 S P}{3}\right)
$$


is linear in $P$ and the Legendre transform is singular:

$$
V=\left.\frac{\partial H}{\partial P}\right|_{S, Q}=\frac{4 \pi}{3}\left(\frac{S}{\pi}\right)^{3 / 2}
$$

is independent of $P$ and so cannot be inverted to obtain $P(V)$. Conversely, when $J$ is zero, the Legendre transform of (23) is

$$
U=\frac{1}{2} \sqrt{\frac{S}{\pi}}\left(1+\frac{\pi Q^{2}}{S}\right),
$$

which is independent of the volume and is not equal to the $J \rightarrow 0$ limit of (22), unless a constraint, $\left(\frac{3 V}{4 \pi}\right)^{2}=\left(\frac{S}{\pi}\right)^{3}$, is imposed. Indeed (251) gives the wrong $J=0$ temperature (unless $Q=P=0$, in which case $U=H$ and $T=\frac{\partial U}{\partial S}=\frac{1}{4 \pi r_{+}}$is the correct Hawking temperature for a Schwarzschild black hole). To get the correct temperature from (221) in the $J \rightarrow 0$ limit we must take the partial derivative with respect to $S$ before setting $J$ to zero, and take note of the fact that $\left(\frac{3 V}{4 \pi}\right)^{2}=\left(\frac{S}{\pi}\right)^{3}$ when $J=0$. This constraint can be derived from (22) directly by observing that $U$ is not differentiable with respect to $J$ at $J=0$ unless $\left(\left(\frac{3 V}{4 \pi}\right)^{2}-\left(\frac{S}{\pi}\right)^{3}\right)\left(J^{2}+\frac{S Q^{2}}{\pi}\right)$ vanishes there. It can also be seen directly when $a=0$ in (13) and (17).

To derive the relation between the pressure and the volume in general we first define $v:=\frac{3 V}{4 \pi}$ and $s:=\frac{S}{\pi}$. Then (22) becomes

$$
U(s, v, J, Q)=\frac{1}{s^{3}}\left\{\frac{v}{2}\left(s^{2}+s Q^{2}+2 J^{2}\right)-|J| \sqrt{\left(v^{2}-s^{3}\right)\left(J^{2}+s Q^{2}\right)}\right\}
$$

with temperature

$$
T=\frac{1}{\pi} \frac{\partial U}{\partial s}=\frac{|J|\left\{3 J^{2}\left(2 v^{2}-s^{3}\right)+s Q^{2}\left(5 v^{2}-2 s^{3}\right)\right\}}{2 \pi s^{4} \sqrt{\left(v^{2}-s^{3}\right)\left(J^{2}+s Q^{2}\right)}}-\frac{v\left(s^{2}+2 s Q^{2}+6 J^{2}\right)}{2 \pi s^{4}}
$$

and pressure

$$
P=-\frac{3}{4 \pi} \frac{\partial U}{\partial v}=\frac{3 v|J|}{4 \pi s^{3}} \sqrt{\frac{J^{2}+s Q^{2}}{v^{2}-s^{3}}}-\frac{3}{8 \pi s^{3}}\left(s^{2}+s Q^{2}+2 J^{2}\right) .
$$

In the $J \rightarrow 0$ limit $|J|$ and $\sqrt{v^{2}-s^{3}}$ must vanish together for finite $T$ and $P$. The equation of state, in the form of the relation between the pressure, 
the temperature and the volume, can be obtained by eliminating $s$ between (27) and (28). In the limit $J \rightarrow 0$ the temperature is

$$
T_{J \rightarrow 0}=\frac{8 \pi v^{4 / 3} P+v^{2 / 3}-Q^{2}}{4 \pi v},
$$

which is the correct temperature as a function of $P, Q$ and $s$, as can be seen from (14), with $a=0$, using (11), (13), (17) and $J=a M$. When $Q=0$ (29) reduces to the equation of state in [2].

The small $J$ correction to (29) is

$$
T=\frac{8 \pi P v^{2}+v^{4 / 3}-Q^{2} v^{2 / 3}-6 J^{2}}{4 \pi v^{5 / 3}}+O\left(\frac{J^{4}}{v^{9 / 3}}, \frac{J^{2} Q^{2}}{v^{9 / 3}}\right) .
$$

The angular momentum term dominates at small volumes for any $J>0$. The first few terms in an expansion for the pressure are

$$
P=\frac{T}{2 v^{1 / 3}}-\frac{1}{8 \pi v^{2 / 3}}+\frac{Q^{2}}{8 \pi v^{4 / 3}}+\frac{3 J^{2}}{4 \pi v^{2}}+O\left(\frac{J^{4}}{v^{10 / 3}}, \frac{J^{2} Q^{2}}{v^{10 / 3}}\right)
$$

(the virial expansion for $Q=0$ is developed in more detail in the second appendix.)

The $P-V$ diagram is plotted to this order in figure 1 with $Q=0$ and $J=1$ (for clarity the horizontal axis in figure 1 is $v^{1 / 3}$ rather than $v$ ). There is a critical point at $T_{c} \approx 0.0413, P_{c} \approx 0.00280$ and $v_{c}^{1 / 3} \approx 3.08$. This critical point represents a second order phase transition, similar in nature to that of a van der Waals gas - it is the same critical point that was found in [9] by fixing the pressure and varying $J$ (the authors of [9] use $P=\frac{3}{8 \pi}=0.119$, corresponding to $L=1$, and find a critical value of $J$ at $J_{c}=0.0236$ giving $\frac{3 J_{c}}{8 \pi}=0.00282$ ). The corrections in equation (31) become large when $v$ is very small, but at the critical point the error in the pressure is of order $\sim \frac{1}{v^{10 / 3}} \sim 10^{-5}$, or about $1 \%$ of $P_{c}$. When $v^{1 / 3} \lesssim 2$ the corrections are of the same order as $P$ and the virial expansion breaks down. In the limit when $v$ is small relative to $J$, a large $J$ expansion shows that the leading order behaviour is $P \approx \frac{3 J^{2}}{8 \pi v^{2}}$, which is the same low $v$ behaviour as figure 1 but reduced by a factor 2 .

Thus by taking the $J \rightarrow 0$ limit carefully the temperature can be kept finite when $v^{2} \rightarrow s^{2}$, but the non-analyticity in (22) is still present in the second derivative with respect to $S$, which diverges when $J=0$ and $v^{2}=$ 
$s^{3}$. This has the important consequence that the heat capacity at constant volume, $C_{V}=T /\left(\frac{\partial T}{\partial S}\right)_{V, J, Q}$, tends to zero when $J=0$, as observed in [2]. $C_{V}$ can be non-zero for $J \neq 0$, and it is plotted in figure 2 for $Q=0$ as a function of $S$ and $J$, with $V=1$. For comparison the heat capacity at constant pressure, $C_{P}=T /\left(\frac{\partial T}{\partial S}\right)_{P, J, Q}$, is plotted in figure 3 for $Q=0$ as a function of $S$ and $J$, with $P=1 . C_{P}$ vanishes when $T=0$ and diverges when $\frac{\partial T}{\partial S}=0$.

A full stability analysis was carried out in [9] and figure 4 shows the phase diagram, when $Q=0$, in terms of $S / L^{2}$ and $J / L^{2}$. The top curve, labelled I, is the $T=0$ limit, the region above and to the left of this curve is unphysical because $T<0$ there. $C_{P}$ in figure 3 vanishes on curve I. Curve II is the curve on which $\Omega=1 / L$, below which the black hole can be in equilibrium with radiation rotating at infinity [15]. On this curve asymptotic spacetime is conformal to the $2+1$ dimensional Einstein universe rotating at the speed of light, the region below and to the right of this curve is of relevance to conformal field theories. Curve III is the curve marking the edge of local stability, the determinant of the Hessian of the Gibbs free energy is divergent on this curve. The heat capacity, $C_{P}$, diverges on curve IV, which is blown up in the right hand picture (this curve was not included in the analysis of reference [9]). Curve V marks the Hawking-Page phase transition, below this curve the black hole is globally stable. The three curves marking the Hawking-Page transition, the edge of local stability and the $\Omega=1 / L$ curve all have similar asymptotic forms for large $S / L^{2}, \frac{J}{L^{2}} \approx \frac{S^{2}}{2 \pi^{2} L^{4}}+O\left(\frac{S}{L^{2}}\right)$.

In the next section it will be more convenient to use the variables $S, P$, $J$ and $Q$, rather than $S, V, J$ and $Q$, in terms of which

$$
U=\frac{\left(S+\pi Q^{2}\right)\left(S+\pi Q^{2}+\frac{8 P S^{2}}{3}\right)+4 \pi^{2}\left(1+\frac{4 P S}{3}\right) J^{2}}{2 \sqrt{\pi S\left[\left(S+\pi Q^{2}+\frac{8 P S^{2}}{3}\right)^{2}+4 \pi^{2}\left(1+\frac{8 P S}{3}\right) J^{2}\right]}},
$$

which is manifestly positive. In terms of geometrical variables,

$$
U=\frac{\left(r_{+}^{2}+a^{2}\right)\left(2 L^{4}+a^{2} r_{+}^{2}-a^{2} L^{2}\right)+q^{2} L^{2}\left(2 L^{2}-a^{2}\right)}{4 L^{4} \Xi^{2} r_{+}} .
$$




\section{$3 \quad$ Extracting energy from rotating black holes}

For an electrically neutral black hole, we set $Q=0$ in (32) and the internal energy $U=H-P V$, written in terms of $S, L^{2}=\frac{3}{8 \pi P}$ and $J$, is

$$
U=\frac{S^{2}\left(1+\frac{S}{\pi L^{2}}\right)+4 \pi^{2} J^{2}\left(1+\frac{S}{2 \pi L^{2}}\right)}{2 \sqrt{\pi S\left(1+\frac{S}{\pi L^{2}}\right)\left[S^{2}\left(1+\frac{S}{\pi L^{2}}\right)+4 \pi^{2} J^{2}\right]}} .
$$

In an isentropic, isobaric process the black hole can yield mechanical work by decreasing the angular momentum. If $J$ is reduced from some finite value to zero the efficiency is

$$
\begin{aligned}
\eta= & \frac{U(J)-U(0)}{H(J)} \\
= & \frac{S^{2}\left(1+\frac{S}{\pi L^{2}}\right)+4 \pi^{2}\left(1+\frac{S}{2 \pi L^{2}}\right) J^{2}}{\left(1+\frac{S}{\pi L^{2}}\right)\left[S^{2}\left(1+\frac{S}{\pi L^{2}}\right)+4 \pi^{2} J^{2}\right]} \\
& -\frac{S}{\sqrt{\left(1+\frac{S}{\pi L^{2}}\right)\left[S^{2}\left(1+\frac{S}{\pi L^{2}}\right)+4 \pi^{2} J^{2}\right]}} .
\end{aligned}
$$

For a given $S$ and $L$ there is a maximal value of $J$ determined by demanding that $T>0$, which requires

$$
J^{2}<\frac{S^{2}}{4 \pi^{2}}\left(1+\frac{S}{\pi L^{2}}\right)\left(1+\frac{3 S}{\pi L^{2}}\right) .
$$

The greatest efficiency is for extremal black holes, when the bound (36) is saturated,

$$
\eta=\frac{\left(4 \pi L^{2}+3 S\right)}{2\left(2 \pi L^{2}+3 S\right)}-\frac{\pi L^{2}}{\left(\pi L^{2}+S\right) \sqrt{2 \pi L^{2}+3 S}} .
$$

The asymptotically flat case $(\Lambda=0)$ is obtained by taking the limit $L \rightarrow \infty$, $\eta=1-\frac{1}{\sqrt{2}}=0.29 \ldots$, which is the familiar result [16]. For finite $L$ the efficiency is greater than the asymptotically flat value. It has a maximum value of $0.5184 \ldots$ when $S / L^{2}=15.39 \ldots$ (obtained by solving a quartic equation for $S$ ), and asymptotes to 0.5 for large $S$.

Differentiating (19) with respect to $P$, we see that the thermodynamic volume is a monotonically increasing function of $J$, hence the volume decreases as $J$ is lowered, keeping the area constant. The black hole also heats up during the process. 
When the black hole is charged there are various possibilities for extracting work through an isentropic, isobaric process: one could decrease $J$ keeping $Q$ constant or decrease $Q$ keeping $J$ constant or decrease both simultaneously. Since all that matters is the initial and final values of $U$, the initial and final values of $J$ and $Q$ uniquely determine the efficiency in such a process.

If both $J$ and $Q$ are both non-zero initially and are both decreased to zero, the efficiency is

$$
\begin{array}{r}
\eta=\frac{\left(S+\pi Q^{2}\right)\left(S+\pi Q^{2}+\frac{S^{2}}{\pi L^{2}}\right)+4 \pi^{2}\left(1+\frac{S}{2 \pi L^{2}}\right) J^{2}}{\left(S+\pi Q^{2}+\frac{S^{2}}{\pi L^{2}}\right)^{2}+4 \pi^{2}\left(1+\frac{S}{\pi L^{2}}\right) J^{2}} \\
-\frac{S}{\sqrt{\left(S+\pi Q^{2}+\frac{S^{2}}{\pi L^{2}}\right)^{2}+4 \pi^{2}\left(1+\frac{S}{\pi L^{2}}\right) J^{2}}} .
\end{array}
$$

The optimal efficiency is for extremal black holes and the extremal value of $J$ is given by

$$
J_{\max }^{2}=\frac{1}{4}\left[\left(\frac{S}{\pi}\right)^{2}\left(1+\frac{S}{\pi L^{2}}\right)\left(1+\frac{3 S}{\pi L^{2}}\right)+2\left(\frac{S}{\pi}\right)^{2}\left(\frac{Q}{L}\right)^{2}-Q^{4}\right],
$$

when $T$ in (20) vanishes. Positivity of $J_{\text {max }}^{2}$ then imposes a restriction on the allowed range of $Q^{2}$,

$$
0 \leq Q^{2} \leq\left(\frac{S}{\pi}\right)\left(1+\frac{3 S}{\pi L^{2}}\right)
$$

The greatest efficiency is for an extremal black hole with maximal charge,

$$
Q_{\max }^{2}=\left(\frac{S}{\pi}\right)\left(1+\frac{3 S}{\pi L^{2}}\right)
$$

when

$$
\eta=\frac{\pi L^{2}+3 S}{2\left(\pi L^{2}+2 S\right)} .
$$

For large $S$ efficiencies of up to $75 \%$ are possible in principle for extremal charged, rotating AdS black holes, though as we saw in the previous section this is reduced to $51.8 \%$ for extremal rotating, but electrically neutral, black holes. For comparison, the equivalent figures in asymptotically flat spacetime, with $\Lambda=0$, are $50 \%$ and $29.3 \%$ respectively. 


\section{Conclusions}

The thermodynamics of rotating black holes in four dimensional in antide Sitter space-time has been discussed in detail, with particular attention payed to the rôle of pressure and the volume. The negative cosmological constant is interpreted as a positive pressure and treated as a thermodynamic variable whose conjugate thermodynamic variable is a thermodynamic volume. For rotating black holes the thermodynamic volume is independent of the geometric area, and hence independent of the entropy, allowing a full thermodynamic treatment.

The black hole mass is associated with the enthalpy $H$, as suggested in [1], and the internal energy $U=H-P V$ is lowered relative to the mass by a positive pressure. Vanishing angular momentum is a non-analytic point of the thermodynamics and must be treated with some care, in particular one cannot fully understand black hole thermodynamics simply by focusing on the non-rotating, Schwarzschild case - rotation is essential for a complete analysis.

The black hole equation of state has been analysed in terms of pressure and volume and a virial expansion developed. Non-zero angular momentum causes a rapid rise in pressure as the volume is reduced at constant temperature, a rise that is not present at zero angular momentum. The critical point found in [9] is found to be very similar in form to that of a van der Waals gas.

In a Penrose process the volume decreases as the black hole looses angular momentum and the $P d V$ term in the first law reduces the amount of energy available to do work. However a negative cosmological constant also extends the range of the angular momentum, pushing the maximum allowed value beyond that of the $\Lambda=0$ case, so that in fact more work can be extracted from an extremal black hole in a space-time that is asymptotically anti-de Sitter than from one in a space-time that is asymptotically flat. Efficiencies of up to $75 \%$ are theoretically achievable for a charged black hole in asymptotically anti-de Sitter space-time, compared to $50 \%$ in asymptotically flat space-time. For electrically neutral black holes the corresponding figures are $51.8 \%$ compared to $29.3 \%$.

The analysis here has focused on the $\Lambda<0$ case for three reasons. Firstly there is an ambiguity in the definition of thermodynamic variables in the $\Lambda>0$ case, there are two relevant event horizons to be considered and hence two different temperatures in general. For a general choice of black hole 
parameters it is not possible to make the Euclidean geometry regular by a unique periodic identification of Euclidean time. Secondly $\Lambda>0$ implies negative pressure and consequent thermodynamic instabilities. This latter problem is not necessarily a fatal objection, black holes are well known to be unstable for $\Lambda=0$ because the heat capacity, more precisely $C_{P}$, is negative, but we can still consider metastable situations in which a temperature can be defined for a period of time much shorter than the timescale of thermal instability. Indeed there are regions of parameter space for $\Lambda<0$ in which $C_{P}<0$, and we can still learn something about physical properties of black holes in this regime. Negative pressures can be useful in metastable situations [17]. Lastly it is not even clear how to define the mass of a black hole in a $\Lambda>0$ space-time as there is no accessible asymptotic regime in which one can compare with the zero mass solution.

\section{Appendix 1}

To calculate the internal energy $U=H-P V$, we write the enthalpy in the form

$$
H=\sqrt{\alpha+\beta P+\gamma P^{2}},
$$

where

$$
\begin{aligned}
\alpha & :=\frac{\pi}{S}\left\{\frac{1}{4}\left(\frac{S}{\pi}+Q^{2}\right)^{2}+J^{2}\right\} \\
\beta & :=\frac{4 \pi}{3}\left\{\frac{S}{\pi}\left(\frac{S}{\pi}+Q^{2}\right)+2 J^{2}\right\} \\
\gamma & :=\left(\frac{4 \pi}{3}\right)^{2}\left(\frac{S}{\pi}\right)^{3} .
\end{aligned}
$$

Note that the discriminant,

$$
\beta^{2}-4 \alpha \gamma=\frac{64 \pi^{2}}{9} J^{2}\left(J^{2}+\frac{S Q^{2}}{\pi}\right)
$$

is positive.

Now

$$
V=\left.\frac{\partial H}{\partial P}\right|_{S, J, Q}=\frac{\beta+2 \gamma P}{2 H} \quad \Rightarrow \quad P=\frac{2 H V-\beta}{2 \gamma}
$$


This allows us to re-express $H$ as a function of $V$,

$$
H=\frac{1}{2} \sqrt{\frac{\beta^{2}-4 \alpha \gamma}{V^{2}-\gamma}} .
$$

We can immediately conclude that

$$
V^{2}>\left(\frac{4 \pi}{3}\right)^{2}\left(\frac{S}{\pi}\right)^{3}
$$

in agreement with the observation in [13].

It is now straightforward to determine

$$
U=H-P V=H-\left(\frac{H V^{2}}{\gamma}-\frac{\beta V}{2 \gamma}\right)=\frac{\beta V}{2 \gamma}-\frac{\sqrt{\left(V^{2}-\gamma\right)\left(\beta^{2}-4 \alpha \gamma\right)}}{2 \gamma},
$$

which immediately gives (22) in the text.

\section{Appendix 2}

In this appendix the virial expansion is developed. For simplicity we set $Q=0$, but the same techniques can be applied to the case of non-zero $Q$. To develop the expansion we use dimensionless variables

$$
y:=\frac{v}{J^{3 / 2}}, \quad x:=\frac{s}{J}, \quad p:=\frac{8 \pi P J}{3}, \quad t:=2 \pi T J^{1 / 2},
$$

in terms of which equations (27) and (28) can be written

$$
\begin{aligned}
& t=\frac{3\left(2 y^{2}-x^{3}\right)}{x^{4} \sqrt{y^{2}-x^{3}}}-\frac{y\left(x^{2}+6\right)}{x^{4}} \\
& p=\frac{2 y}{x^{3} \sqrt{y^{2}-x^{3}}}-\frac{x^{2}+2}{x^{3}} .
\end{aligned}
$$

When $y$ and $x$ are large let $y^{2} \approx x^{3}$, with $y^{2}-x^{3}=z^{2}$, then $t$ is finite provided $z \approx \frac{3}{y^{2 / 3} t}$ in which case $p \approx \frac{2 t}{3 y^{1 / 3}}$. Replacing $x$ with $z$ in equations (51) and (52) gives

$$
\begin{aligned}
3\left(y^{2}+z^{2}\right) & =\left\{t\left(y^{2}-z^{2}\right)^{4 / 3}+y\left(y^{2}-z^{2}\right)^{2 / 3}+6 y\right\} z \\
\left(y^{2}-z^{2}\right) z p & =2 y-z\left\{\left(y^{2}-z^{2}\right)^{2 / 3}+2\right\} \\
\Rightarrow \quad p & =\frac{2}{z(y+z)}-\frac{1}{\left(y^{2}-z^{2}\right)^{1 / 3}} .
\end{aligned}
$$


Now we expand in powers of $u=\frac{1}{y^{1 / 3}}$. Let $z=\frac{3 u^{2}}{t} \zeta$ for some $\zeta(t, u)$, in terms of which (51) becomes

$$
t\left(1+\frac{9 u^{10} \zeta^{2}}{t^{2}}\right)=\left\{t\left(1-\frac{9 u^{10} \zeta^{2}}{t^{2}}\right)^{4 / 3}+u\left(1-\frac{9 u^{10} \zeta^{2}}{t^{2}}\right)^{2 / 3}+6 u^{5}\right\} \zeta,
$$

from which we can immediately conclude that

$$
\zeta=\frac{t}{t+u+6 u^{5}}+\omega(t, u)
$$

where an expansion of $\omega$ in $u$ starts at order 10. Any desired order can be obtained by further expanding $\omega$

$$
\omega=u^{10} \sum_{n=0}^{\infty} a_{n}(t) u^{n}
$$

with the co-efficients $a_{n}(t)$ to be determined. Putting (57) and (56) into (55), and equating co-efficients of powers of $u$, gives an iterative procedure for evaluating the co-efficients $a_{n}$ which can then be used to show that:

$$
\omega=7 \frac{u^{10}}{t^{2}}-16 \frac{u^{11}}{t^{3}}+27 \frac{u^{12}}{t^{4}}-40 \frac{u^{13}}{t^{5}}+55 \frac{u^{14}}{t^{6}}-36 \frac{2+3 t^{4}}{t^{7}} u^{15}+O\left(u^{16}\right) .
$$

Finally using this expansion in (54) gives the required virial expansion

$$
\begin{array}{r}
p=\frac{2 t u}{3}-\frac{1}{3} u^{2}+2 u^{6}-u^{10}\left(8 \frac{u}{t}-9 \frac{u^{2}}{t^{2}}+10 \frac{u^{3}}{t^{3}}-11 \frac{u^{4}}{t^{4}}+12 \frac{u^{5}}{t^{5}}\right. \\
\left.-13 \frac{1+6 t^{4}}{t^{6}} u^{6}\right)+O\left(u^{17}\right),
\end{array}
$$

the first three terms of which are used in the text.

\section{References}

[1] D. Kastor, S. Ray and J. Traschen, Class. Quantum Grav. 26 (2009) 195011, arXiv:0904.2765 [hep-th]].

[2] B.P. Dolan, Class. Quantum Grav. 28 (2011) 125020, arXiv:1008.5023[gr-qc]]. 
[3] M. Henneaux and C. Teitelboim, Phys. Lett. 143B (1984) 415;

M. Henneaux and C. Teitelboim, Phys. Lett. 222B (1989) 195.

[4] C. Teitelboim, Phys. Lett. 158B (1985) 293.

[5] S. Wang, S-Q. Wu, F. Xie and L. Dan, Chin. Phys. Lett., 23 (2006) 1096, arXiv:hep-th/0601147.

[6] Y. Sekiwa, Phys. Rev. D73 (2006) 084009, arXiv:hep-th/0602269].

[7] E.A. Larrañaga Rubio, Stringy Generalization of the First Law of Thermodynamics for Rotating BTZ Black Hole with a Cosmological Constant as State Parameter, arXiv:0711.0012 [gr-qc]].

[8] S. Wang, S-Q. Wu, F. Xie and L. Dan, Chin. Phys. Lett., 23 (2006) 1096, arXiv:hep-th/0601147;

S. Wang, Thermodynamics of high dimensional Schwarzschild de Sitter spacetimes: variable cosmological constant, arXiv:gr-qc/0606109.

[9] M.M. Caldarelli, G. Cognola and D. Klemm, Class. Quantum Grav. 17 (2000) 399, arXiv:hep-th/9908022.

[10] B. Carter, Comm. Math. Phys. 10 (1968) 280.

[11] M. Henneaux and C. Teitelboim, Comm. Math. Phys. 98 (1985) 391.

[12] G.W. Gibbons, M.J. Perry and C.N. Pope, Class. Quantum Grav. 22 (2005) 1503, arXiv:hep-th/0408217.

[13] M. Cvetic, G.W. Gibbons, D. Kubiznak and C.N. Pope, Black Hole Enthalpy and an Entropy Inequality for the Thermodynamic Volume, arXiv:1012.2888[gr-qc]].

[14] D. Christodoulou, Phys. Rev. Lett. 25 (1970) 1596;

D. Christodoulou and R. Ruffini, Phys. Rev. D4 (1971) 3552.

[15] S.W. Hawking, C.J. Hunter and M.M. Taylor-Robinson, Phys. Rev. D59 (1999) 0640055, arXiv:hep-th/9811056.

[16] R.M. Wald, General Relativity, University of Chicago Press (1984).

[17] See e.g. page 44 in L.D. Landau and E.M. Lifschitz, Statistical Physics. Part 1, 3rd ed. (1980) Elsevier. 


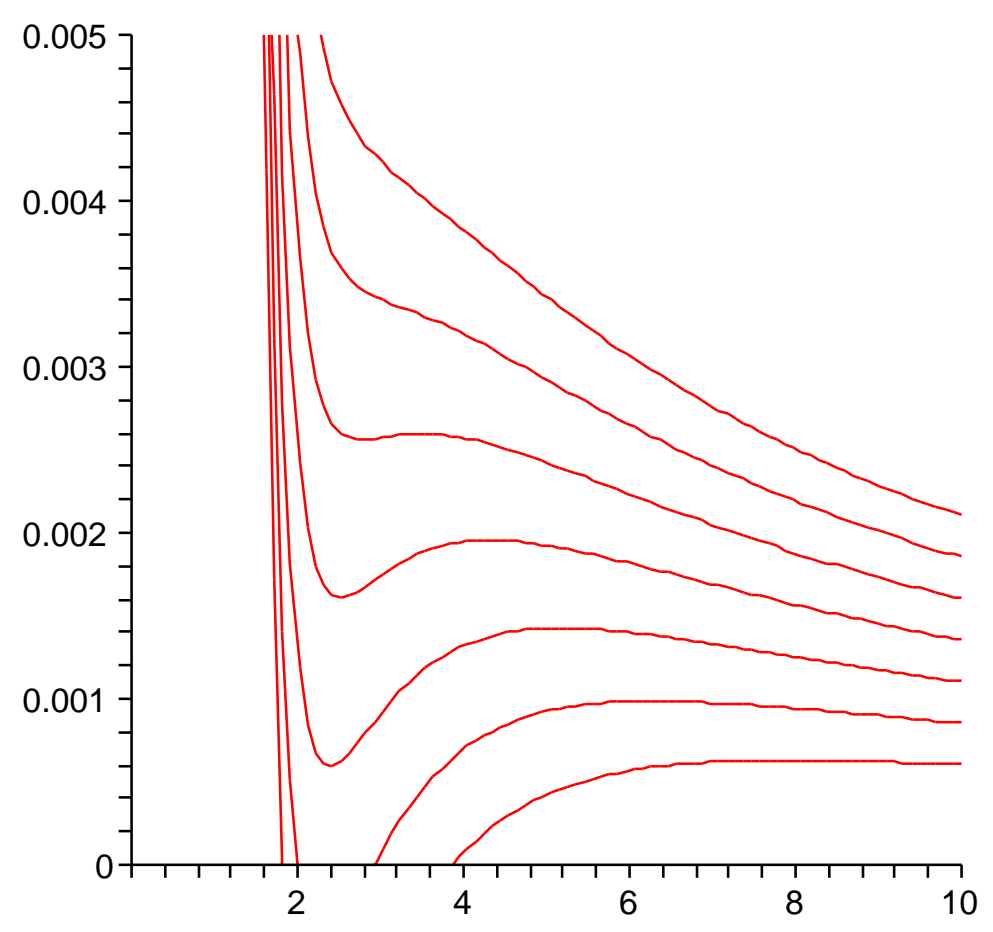

Figure 1: $P-V$ diagram for $J=1$ and $Q=0$, plotted using the approximation in equation (31). $P$ is plotted as a function of $v^{\frac{1}{3}}$ for $T=0.02,0.025,0.3$, $0.35,0.04,0.045$ and 0.05 . The critical point for this value of $J$ is $T_{c} \approx 0.0413$, $P_{c} \approx 0.00280$ and $v_{c} \approx 3.08 . J>0$ causes the rapid rise in $P$ at low values of $v$. 


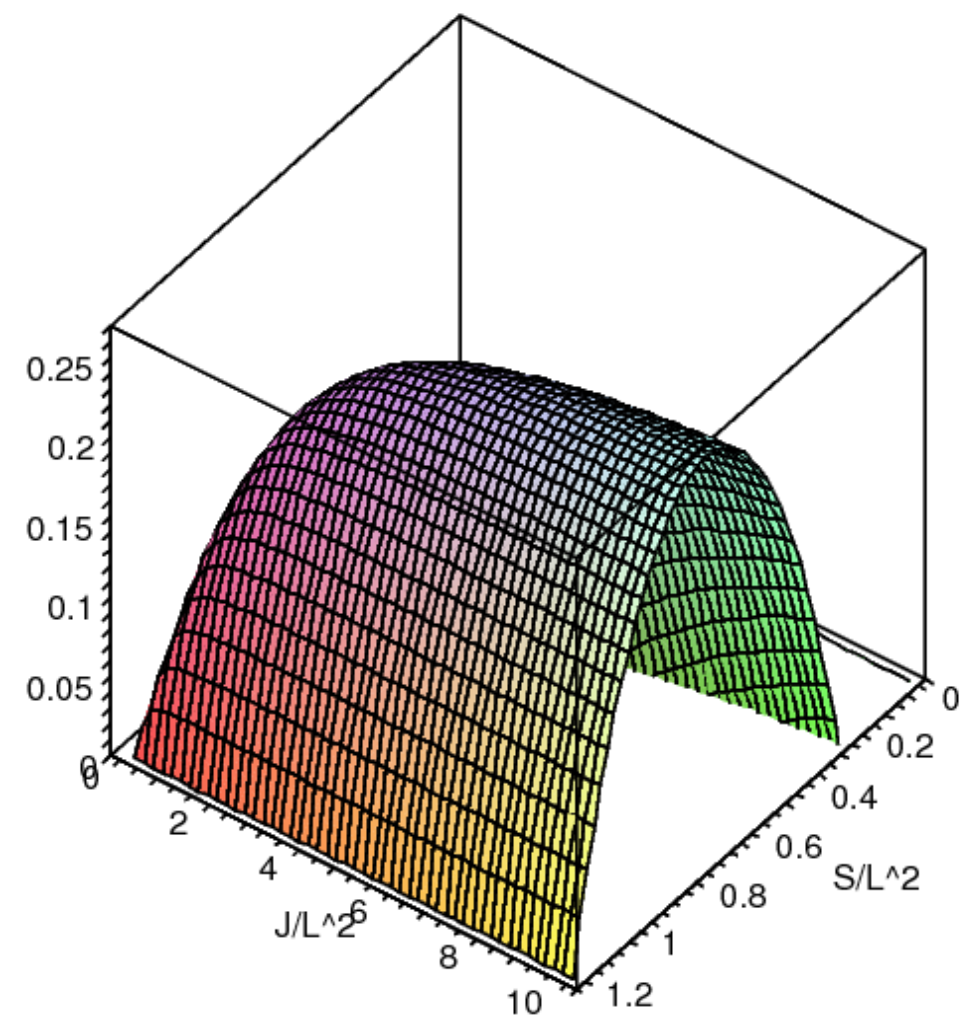

Figure 2: $C_{V}$ as a function of $S / L^{2}$ and $J / L^{2}$, with fixed volume set to $L^{3}$. 


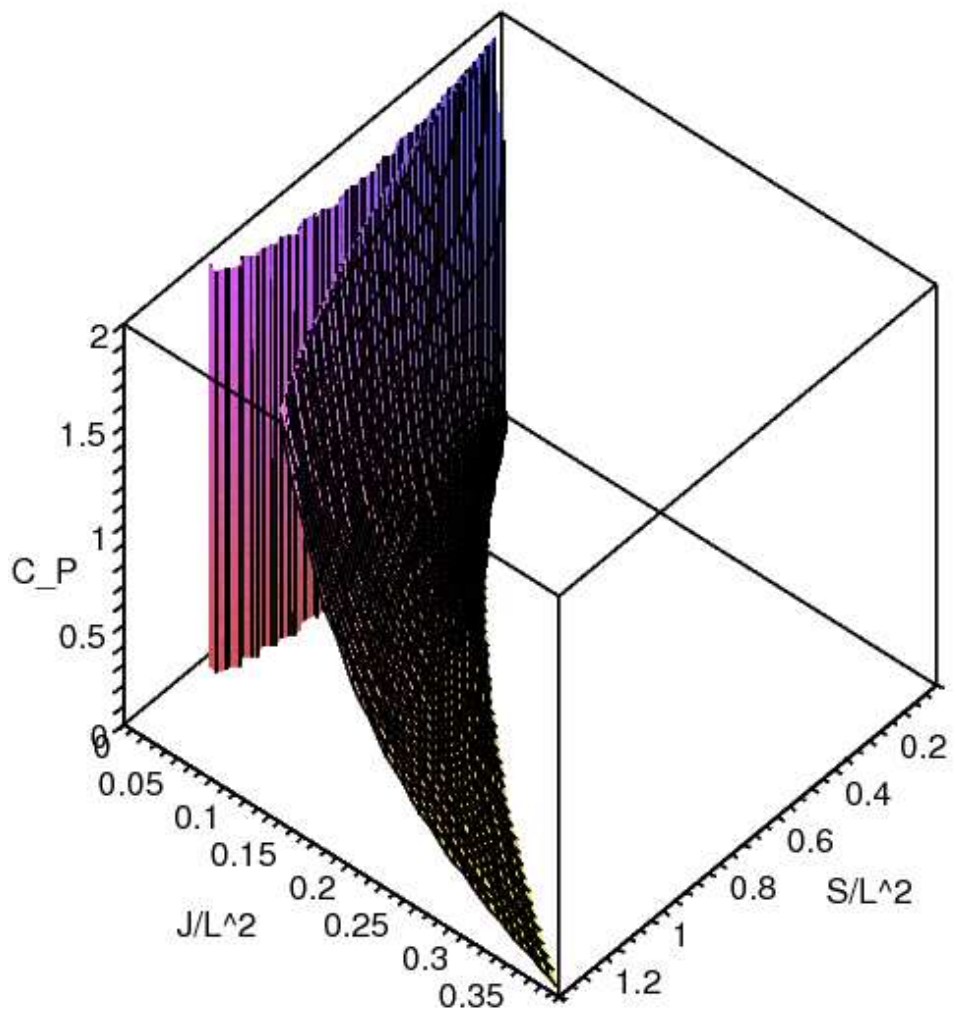

Figure 3: $C_{P}$ as a function of $S / L^{2}$ and $J / L^{2}$, with fixed pressure set to $\frac{3}{8 \pi}$, i.e $L=1$. $C_{P}$ diverges along curve IV in figure 4 and vanishes along curve I. 

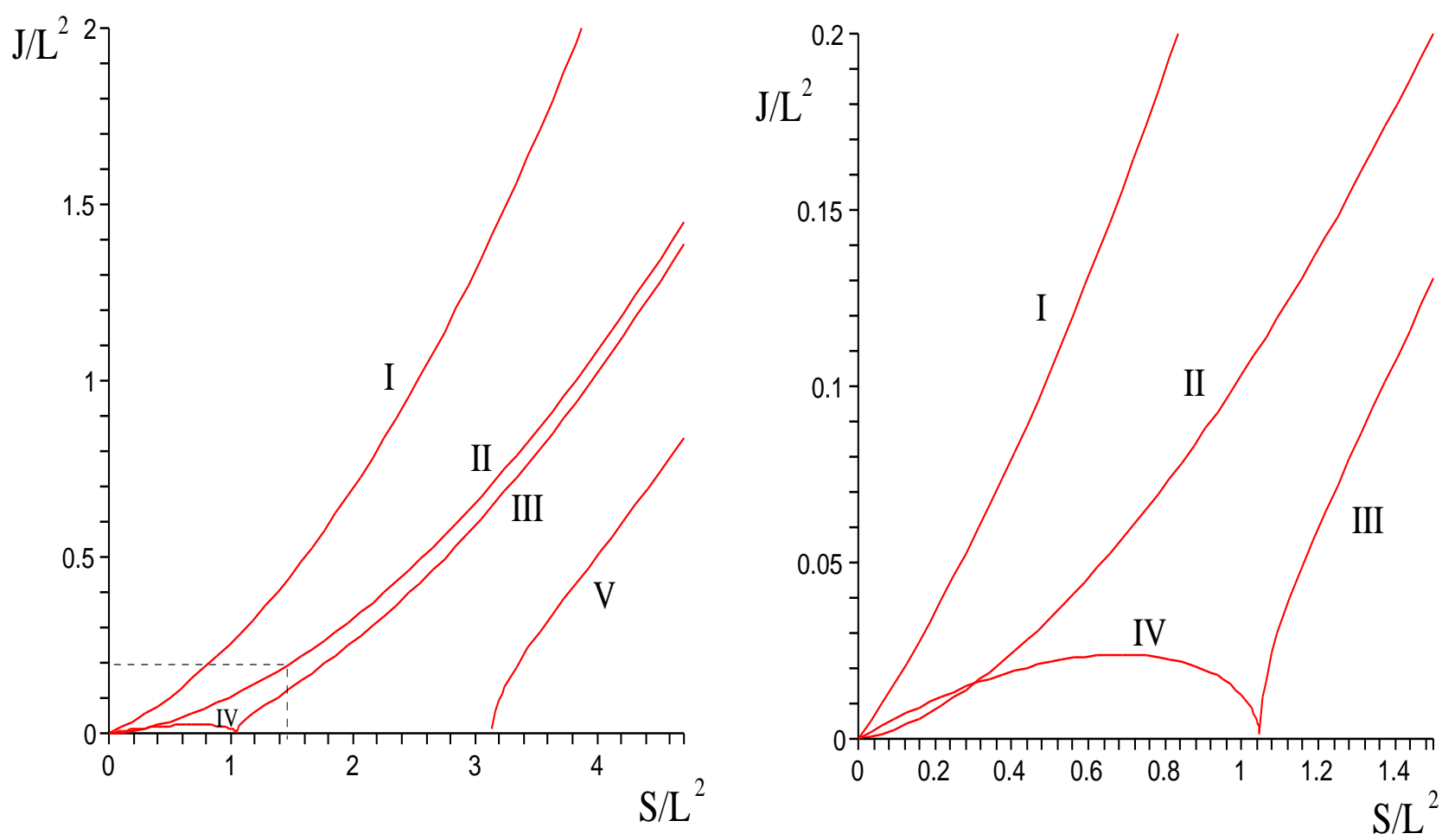

Figure 4: phase diagram for $q=0$, plotted in terms of $S / L^{2}$ and $J / L^{2}$.

The region above $\mathrm{I}$ is forbidden, because $T<0$;

in the region above curve II the 3-d Einstein universe at infinity rotates faster than the speed of light;

curve III bounds the region of local stability (an analysis of the Gibbs free energy shows that the black hole is locally unstable above curve III);

$C_{P}$ diverges on curve IV;

in the region above curve $\mathrm{V}$ the black hole is unstable due to the HawkingPage phase transition;

in region below curve $\mathrm{V}$ the black hole is stable. 